\title{
THE GRAPH OF EQUIVALENCE CLASSES OF ZERO-DIVISORS OF A POSET
}

\author{
HONGXING LIU \\ Shandong Normal University, P. R. China
}

\begin{abstract}
In this paper, we give the definition of the graph of equivalence classes of zero-divisors of a poset $P$. We prove that if $[a]$ has maximal degree in $V\left(\Gamma_{E}(P)\right)$, then $\operatorname{ann}(a)$ is maximal in $\operatorname{Anih}(P)$. Also, we give some other properties of the graph $\Gamma_{E}(P)$. Moreover, we characterize the cut vertices of $\Gamma_{E}(P)$ and study the cliques of these graphs.
\end{abstract}

\section{INTRODUCTION}

The concept of zero-divisor graph was first introduced by Beck in [7] to investigate the interplay between ring-theoretic properties and graph-theoretic properties. The concept of zero-divisor graph has also been extended to many algebraic structures such as rings, semigroups, semirings (see [4-11,16]). Halaš and Jukl ([13]) introduced the zero-divisor graph of a poset. Since then, many authors continued to study the zero-divisor graphs of posets, see $[1,15,16,20]$. Let $R$ be a ring and $r, s \in R$. Define $r \sim s$ if and only if $\operatorname{ann}(r)=\operatorname{ann}(s)$. Write $[r]=\{s \in R \mid r \sim s\}$ and $R_{E}=\{[r] \mid r \in R\}$. Denote by $\Gamma_{E}(R)$ the graph of equivalence classes of zero-divisors of $R$. The set of vertices $V\left(\Gamma_{E}(R)\right)$ is $R_{E} \backslash\{[0],[1]\}$ and two vertices are adjacent if and only if $[r][s]=[0]$, if and only if $r s=0$. Motivated by ideas in paper [18], Spiroff and Wickham ([19]) studied the graph of equivalence classes of zero-divisors of a commutative Noetherian ring. Anderson and LaGrange ([2]) continued to study these graphs. In [2], the graph is called the compressed zero-divisor graph. In this paper, we will extend the graph of equivalence classes of zero-divisors to a poset $P$ and study the properties of these graphs.

2010 Mathematics Subject Classification. 05E99, 06A07.

Key words and phrases. Zero-divisor graph, poset, cut vertex, equivalence class, clique. 
The paper is constructed as follows: In Section 2, we give some relevant definitions and notations of graphs and posets. In Section 3, we give the definition of the graph of equivalence classes of zero-divisors of a poset $P$ and study the basic properties of these graphs. In Section 4, we investigate the cut vertices and clique number of the graph $\Gamma_{E}(P)$.

Throughout, all posets $P$ will be a poset with 0 and 1 and all graphs will be simple graphs.

\section{Preliminaries}

Let $(P, \leq)$ be a partially ordered set (abbreviated as a poset) and $X \subseteq P$. Let $L(X)=\{y \in P \mid y \leq x$ for all $x \in X\}$ denote the lower cone of $X$. Dually, let $U(X)=\{y \in P \mid y \geq x$ for all $x \in X\}$ denote the upper cone of $X$. If $X=\left\{x_{1}, \ldots, x_{n}\right\}$, we shall write $L\left(x_{1}, \ldots, x_{n}\right)$ or $U\left(x_{1}, \ldots, x_{n}\right)$ instead of $L(X)$ or $U(X)$.

Let $P$ be a poset and $\emptyset \neq I \subseteq P$. Then $I$ is called an ideal of $P$ if $x \in I$ and $y \leq x$, then $y \in I$. A proper ideal $I$ of $P$ is called prime if for all $x, y \in P, L(x, y) \subseteq I$ implies $x \in I$ or $y \in I$.

For $x \in P$, the set $\operatorname{ann}(x)=\{y \in P \mid L(x, y)=\{0\}\}$ is called the annihilator of $x$.

For $x \in P, x$ is called a zero-divisor of $P$ if there exists $0 \neq y \in P$ such that $L(x, y)=\{0\}$. Denote by $Z(P)$ the zero-divisors of $P$ and write $Z(P)^{\times}=Z(P) \backslash\{0\}$.

The zero-divisor graph of $P$, denoted by $\Gamma(P)$, is as follows: the set of vertices is $V(\Gamma(P))=Z(P)^{\times}$and distinct vertices $x$ and $y$ are adjacent if and only if $L(x, y)=\{0\}([1])$.

Let $G$ be a graph. For $k \geq 2$, a graph is called a $k$-partite graph if the vertices of the graph are partitioned into $k$ disjoint sets such that there is no edge between two vertices in the same set. A 2-partite graph is usually called a bipartite graph. It is well known that a graph is bipartite if and only if it contains no cycle of odd length. A complete bipartite graph is a bipartite graph such that every vertex in one set is connected to every vertex in the other set. The complete graph $K_{n}$ is a graph with $n$ vertices in which each vertex is connected to each of the others. The diameter of a graph $G$ is the largest distance between two vertices in $G$, denoted by $\operatorname{diam}(G)$. A clique of a graph $G$ is a subset of its vertices such that there exists an edge between each pair of vertices in the subset. The clique number $\operatorname{cl}(G)$ of a graph $G$ is the number of vertices in a maximum clique in $G$.

\section{BAsic PROperties of the GRAPH $\Gamma_{E}(P)$}

In this section, we will define the graph of equivalence classes of zerodivisors of a poset $P$ and investigate the properties of this graph. 
An element $0 \neq p$ of a poset $P$ is called an atom if there exists no element $x \in P$ such that $0<x<p$. The set of atoms of $P$ is denoted by $\operatorname{Atom}(P)$. If $p \in P$, set $\operatorname{atom}(p)=\{a \in \operatorname{Atom}(P) \mid a \leq p\}$.

For any elements $a, b \in P$, define a relation on $P$ by $a \sim b$ if and only if $\operatorname{ann}(a)=\operatorname{ann}(b)$. Then $\sim$ is an equivalence relation on $P$.

For any $a \in P$, let $[a]=\{r \in P \mid r \sim a\}$. It is easy to get the following statements.

Lemma 3.1. Let $P$ be a poset. Then:

1) $\operatorname{ann}(1)=\{0\}$ and $\operatorname{ann}(0)=P$. Moreover, if $a \neq 0$, then $[a] \neq[0]$.

2) $[a] \subseteq Z(P)$, for all $a \in P \backslash\{0,1\}$.

Let $\bar{P}=\{[a] \mid a \in P\}$. Define a partial order relation on $\bar{P}$ by $[a] \leq^{\prime}[b]$ if and only if $\operatorname{ann}(b) \subseteq \operatorname{ann}(a)$. It is clear that this partial order relation is well-defined and $\left(\bar{P}, \leq^{\prime}\right)$ is a poset. [0] is the least element in $\bar{P}$ and [1] is the largest element in $\bar{P}$. Without causing confusion, we will let $\leq$ represent the partial order relation on both $P$ and $\bar{P}$ in the following.

Now, we give the definition of the graph of equivalence classes of zerodivisors of a poset $P$.

DeFINITION 3.2. The graph of equivalence classes of zero-divisors of a poset $P$ is the graph $\Gamma_{E}(P)=\Gamma(\bar{P})$ whose vertices are the elements in $\bar{P} \backslash\{[0],[1]\}$, such that two distinct vertices $[a]$ and $[b]$ are adjacent if and only if $L([a],[b])=\{[0]\}$.

Let $P$ be a poset as below. Then one can check that $\operatorname{diam}(\Gamma(P))=2$ while $\operatorname{diam}\left(\Gamma_{E}(P)\right)=1$. The properties of the graph $\Gamma_{E}(P)$ and the graph $\Gamma(P)$ are different.
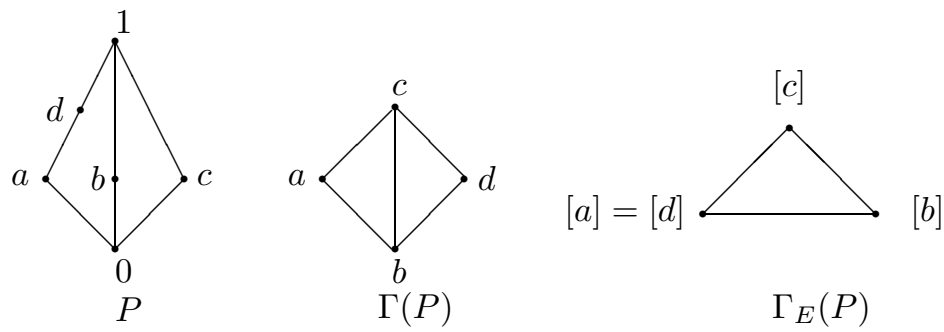

Lemma 3.3. Let $P$ be a poset and $a, b \in P$. Then

1) If $a \leq b$, then ann $(b) \subseteq$ ann $(a)$ and $[a] \leq[b]$ in $\bar{P}$.

2) If $[a] \neq[1],[b] \neq[1]$, and $L([a],[b])=\{[0]\}$, then $L(a, b)=\{0\}$.

3) If $L(a, b)=\{0\}$, then $L([a],[b])=\{[0]\}$.

Proof. 1) Obvious.

2) Suppose $x \in L(a, b)$. Then $x \leq a$ and $x \leq b$. It follows that $\operatorname{ann}(a) \subseteq$ $\operatorname{ann}(x)$ and $\operatorname{ann}(b) \subseteq \operatorname{ann}(x)$. Hence, $[x] \leq[a]$ and $[x] \leq[b]$. Therefore, we have $[x]=[0]$, and so $x=0$ by 1 ) in Lemma 3.1. Hence, $L(a, b)=\{0\}$. 
3) Suppose $[c] \in L([a],[b])$. Then $[c] \leq[a]$ and $[c] \leq[b]$. Hence, $\operatorname{ann}(a) \subseteq$ $\operatorname{ann}(c)$ and $\operatorname{ann}(b) \subseteq \operatorname{ann}(c)$. By $L(a, b)=\{0\}$, we have $b \in \operatorname{ann}(a) \subseteq \operatorname{ann}(c)$, and so $L(b, c)=\{0\}$. Thus $c \in \operatorname{ann}(b) \subseteq \operatorname{ann}(c)$. It follows that $c=0$, and so $[c]=[0]$. Therefore, $L([a],[b])=\{[0]\}$.

Proposition 3.4. Let $P$ be a poset. If $[x]=\left[x_{1}\right]$ and $[y]=\left[y_{1}\right]$, then $L(x, y)=\{0\}$ if and only if $L\left(x_{1}, y_{1}\right)=\{0\}$.

Proof. $\Rightarrow$ : Suppose $[x]=\left[x_{1}\right]$ and $[y]=\left[y_{1}\right]$. Then $\operatorname{ann}(x)=\operatorname{ann}\left(x_{1}\right)$ and $\operatorname{ann}(y)=\operatorname{ann}\left(y_{1}\right)$. Since $L(x, y)=\{0\}$, we have $y \in \operatorname{ann}(x)=\operatorname{ann}\left(x_{1}\right)$, and hence $L\left(x_{1}, y\right)=\{0\}$. That is, $x_{1} \in \operatorname{ann}(y)=\operatorname{ann}\left(y_{1}\right)$. Thus $L\left(x_{1}, y_{1}\right)=$ $\{0\}$.

$\Leftarrow$ : The proof is similar to that of " $\Rightarrow$ ".

Remark 3.5. By Definition 3.2, Lemma 3.3, and Proposition 3.4, we know that the graph $\Gamma_{E}(P)$ is isomorphic to a subgraph of $\Gamma(P)$.

Let $a$ be a vertex of a graph $G$. The degree of $a$ is the number of edge ends at $a$, denoted by $\operatorname{deg}(a)$. Let $N(a)$ be the set of vertices which are adjacent to $a$, then $|N(a)|=\operatorname{deg}(a)$. For any two vertices $u$ and $v$ of a graph $G$, define $u \approx v$ if and only if $N(u)=N(v)$. Let $\Gamma(P)$ be the zero-divisor graph of a poset $P$ and $u, v \in P$. Note that $N(u)=\operatorname{ann}(u) \backslash\{0\}$. Then $u \approx v$ if and only if $\operatorname{ann}(u)=\operatorname{ann}(v)$, if and only if $[u]=[v]$. Let $\bar{u}=\{r \in G \mid r \approx u\}$ and $G / \approx=\{\bar{u} \mid u \in G\}$. Then $G / \approx$ becomes a graph in the natural way with $[u]$ and $[v]$ are adjacent in $G / \approx$ if and only if $u$ and $v$ are adjacent in $G$. Using Lemma 3.3, we get the following analog of [2, Theorem 2.4].

Theorem 3.6. Let $P$ be a poset. Then $\Gamma_{E}(P) \cong \Gamma(P) / \approx$.

Proof. Suppose $a \in P$. Define a map $\varphi: \Gamma_{E}(P) \rightarrow \Gamma(P) / \approx$ by $[a] \mapsto \bar{a}$. By the above comments, the map $\varphi$ is well-defined. One can easily check that $\varphi$ is also bijective. If $[a]-[b]$ is an edge in $\Gamma_{E}(P)$, then $L([a],[b])=\{[0]\}$, and hence $L(a, b)=\{0\}$ by Lemma 3.3. Therefore, $\bar{a}-\bar{b}$ is an edge in $\Gamma(P) / \approx$.

Conversely, if $\bar{a}-\bar{b}$ is an edge in $\Gamma(P) / \approx$, then $a$ and $b$ are adjacent in $\Gamma(P)$, and hence $L(a, b)=\{0\}$. By Lemma 3.3, we get $L([a],[b])=\{[0]\}$. Therefore, $[a]-[b]$ is an edge in $\Gamma_{E}(P)$.

The diameter of the graph $\Gamma_{E}(R)$ is less or equal to 3 , where $R$ is a commutative ring with identity (Proposition 1.4 in [19]). The following statement gives a similar result for the graph $\Gamma_{E}(P)$, where $P$ is a poset.

THEOREm 3.7. Let $P$ be a poset. Then $\Gamma_{E}(P)$ satisfies the following conditions.

1) $\Gamma_{E}(P)$ is connected.

2) $\operatorname{diam}\left(\Gamma_{E}(P)\right) \leq 3$. 
Proof. By the definition of $\Gamma_{E}(P)$, we know that it is also a zero-divisor graph of the poset $\bar{P}$. Using [1, Theorem 3.3], we have that $\Gamma_{E}(P)$ is connected and $\operatorname{diam}\left(\Gamma_{E}(P)\right) \leq 3$.

In [19], Spiroff and Wickham investigated infinite graphs of equivalence classes of zero-divisors of a ring $R$ and associated primes of $R$, where $R$ is a commutative Noetherian ring with identity. We shall study the corresponding problems in poset settings.

Proposition 3.8. Let $P$ be a poset and $a, b \in P$. Then $\operatorname{ann}([a])=\operatorname{ann}([b])$ if and only if $[a]=[b]$.

Proof. $\Rightarrow$ : Let $a, b \in P$ and $\operatorname{ann}([a])=\operatorname{ann}([b])$. Suppose $z \in \operatorname{ann}(a)$. By Lemma 3.3, we have $[z] \in \operatorname{ann}([a])=\operatorname{ann}([b])$, and so $L([z],[b])=\{[0]\}$. Using Lemma 3.3 again, we have $L(z, b)=\{0\}$. This proves that $z \in \operatorname{ann}(b)$, and hence $\operatorname{ann}(a) \subseteq \operatorname{ann}(b)$. Similarly, one can prove that $\operatorname{ann}(b) \subseteq \operatorname{ann}(a)$. Therefore, $[a]=[b]$.

$$
\Leftarrow \text { : Obvious. }
$$

A poset $P$ is atomic if for all $0<b \in P$, there exists an atom $a \in P$ such that $0<a \leq b$. Let $P$ be a poset. Let $\operatorname{Anih}(P)=\{\operatorname{ann}(a) \mid a \in P, \operatorname{ann}(a) \neq$ $P\}$. If $a \in P$ and $\operatorname{ann}(a)$ is maximal among $\operatorname{Anih}(P)$, then $\operatorname{ann}(a)$ is a prime ideal of $P$ ([13], Lemma 2.2).

Proposition 3.9. Let $P$ be a poset. If $a$ is an atom of $P$, then ann $(a)$ is maximal in Anih $(P)$. Moreover, ann $(a)$ is prime. Conversely, if $P$ is atomic and ann $(b)$ is maximal in Anih $(P)$, then there exists an atom a such that $\operatorname{ann}(a)=\operatorname{ann}(b)$.

Proof. Suppose there exists an element $0 \neq c \in P$ with $\operatorname{ann}(a) \subset \operatorname{ann}(c)$. Then there exists $x \in \operatorname{ann}(c) \backslash \operatorname{ann}(a)$, that is, $L(x, c)=\{0\}$, but $L(x, a) \neq\{0\}$. Assume $0 \neq z \in L(x, a)$. Since $a$ is an atom, we must have $z=a$. Hence $a \leq x$. Thus $L(a, c)=\{0\}$, and so $c \in \operatorname{ann}(a)$. Therefore $c \in \operatorname{ann}(c)$. This is impossible. Thus $\operatorname{ann}(a)$ is maximal. By Lemma 2.2 in [13], it follows that $\operatorname{ann}(a)$ is prime.

Conversely, suppose $\operatorname{ann}(b)$ is maximal in $\operatorname{Anih}(P)$ and $a$ is an atom such that $0<a \leq b$. We have $\operatorname{ann}(b) \subseteq \operatorname{ann}(a)$, and so $\operatorname{ann}(b)=\operatorname{ann}(a)$ by the maximality of ann $(b)$.

The following proposition is similar to Proposition 2.2 in [19].

Proposition 3.10. Let $P$ be a poset and $|\operatorname{Atom}(P)|<\infty$. Then $\left|V\left(\Gamma_{E}(P)\right)\right|=\infty$ if and only if there exists $x \in P$ such that ann $(x)$ is maximal in $\operatorname{Anih}(P)$ and $\operatorname{deg}([x])=\infty$.

Proof. $\Rightarrow$ : Suppose Atom $(P)=\left\{a_{1}, a_{2}, \ldots, a_{n}\right\}$. By Proposition 3.9, we know that $\operatorname{ann}\left(a_{1}\right), \operatorname{ann}\left(a_{2}\right), \ldots, \operatorname{ann}\left(a_{n}\right)$ are maximal in $\operatorname{Anih}(P)$. If 
$\operatorname{deg}\left(\left[a_{1}\right]\right)<\infty$, there exist infinitely many vertices $[x]$ such that $L\left([x],\left[a_{1}\right]\right) \neq$ $\{[0]\}$. By Lemma 3.3, we have $L\left(x, a_{1}\right) \neq\{0\}$. If $[v] \neq[x]$ and $L([x],[v])=$ $\{[0]\}$, then $L(x, v)=\{0\} \subseteq \operatorname{ann}\left(a_{1}\right)$. Since ann $\left(a_{1}\right)$ is prime and $x \notin \operatorname{ann}\left(a_{1}\right)$, we have $v \in \operatorname{ann}\left(a_{1}\right)$, and so $[v]$ is adjacent to $\left[a_{1}\right]$. If there exist infinitely many distinct vertices $[v]$ which are adjacent to $\left[a_{1}\right]$, then $\operatorname{deg}\left(\left[a_{1}\right]\right)=\infty$. This is a contradiction. Hence, the set of $[v]$ 's is finite. Note that $[x]$ is adjacent to $[v]$ and the set of $[x]$ 's is infinite. We have $\operatorname{deg}([v])=\infty$ for some $v$. If $\operatorname{ann}(v)$ is maximal, we get the desired result. If $\operatorname{ann}(v) \subseteq \operatorname{ann}\left(a_{i}\right)$ for some $i \neq 1$, we have $\operatorname{deg}\left(\left[a_{i}\right]\right)=\infty$, and we also get the desired result.

$\Leftarrow$ : It is obvious.

Theorem 3.11. Let $P$ be a poset and $a \in P$. If $[a]$ has maximal degree in $V\left(\Gamma_{E}(P)\right)$, then ann $(a)$ is maximal in $\operatorname{Anih}(P)$.

Proof. Suppose $\operatorname{ann}(a) \subseteq \operatorname{ann}(b)$. It is easy to show $N([a]) \subseteq N([b])$. By the maximality of the degree of $[a]$, we have $N([a])=N([b])$. If there exists $z \in \operatorname{ann}(b) \backslash \operatorname{ann}(a)$, by Lemma 3.3 we get $[z]$ is adjacent to $[b]$, but not adjacent to $[a]$. That is, $[z] \in N([b])$, but $[z] \notin N([a])$. This is a contradiction. Therefore, $\operatorname{ann}(a)=\operatorname{ann}(b)$.

The following example proves that the converse of the preceding theorem is not true.

Example 3.12. Let $P_{A}$ be the poset in Figure (A). Then ann $(b)$ is maximal in $\operatorname{Anilh}\left(P_{A}\right)$. One can check that $\operatorname{deg}([b])=2$ and $\operatorname{deg}([a])=3$. Hence, the degree of $[b]$ is not maximal.

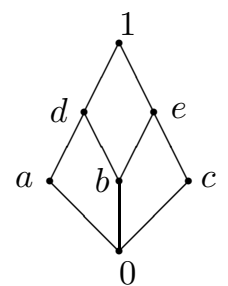

$(A)$

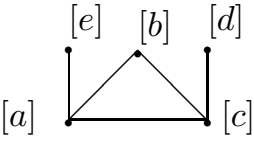

$\Gamma_{E}\left(P_{A}\right)$

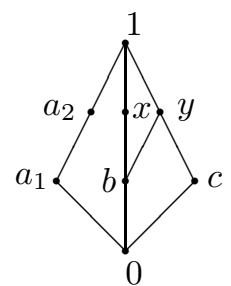

(B)

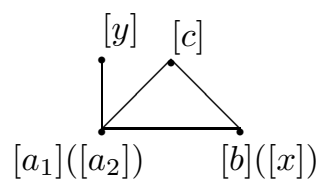

$\Gamma_{E}\left(P_{B}\right)$

4. Cut vertices, Cliques of the graph $\Gamma_{E}(P)$

In this section, we will give a characterization of the cut vertices of the graph $\Gamma_{E}(P)$ and also study the cliques of these graphs.

Let $G$ be a graph. A vertex $a$ is called a cut vertex of $G$ if the removal of $a$ along with edges through $a$ leads to more components than $G$. That is, a vertex $a$ is called a cut vertex if there exist distinct vertices $b$ and $c$ such that $a$ is in every $b-c$ path, where both $b$ and $c$ are different from $a$. Axtell et al. ([6]) studied cut vertices in zero-divisor graphs of commutative rings with identity and proved that if $x$ is a cut vertex of the graph $\Gamma(R)$, then 
the annihilator of $x$ is properly maximal (see [6, Proposition 2.7]). In the following, we investigate cut vertices in the graph $\Gamma_{E}(P)$.

Proposition 4.1. Let $P$ be a poset. If $[a]$ is a cut vertex in $\Gamma_{E}(P)$, then $[a]$ is an atom in $\bar{P}$.

Proof. Suppose $[x]-[a]$ is an edge in $\Gamma_{E}(P)$ and $[0] \neq[b]<[a]$. Then $[x]-[b]$ is also an edge in $\Gamma_{E}(P)$. Using this fact, one can prove that if $[a]$ is not an atom in $\bar{P}$, then $[a]$ is not a cut vertex in $\Gamma_{E}(P)$.

Let $P$ be a poset and $0 \neq x, 0 \neq y \in P$. By Lemma 3.3, $[x]-[y]$ is an edge in $\Gamma_{E}(P)$ if and only if $x-y$ is an edge in $\Gamma(P)$. Hence, we have the following lemma.

Lemma 4.2. Let $P$ be a poset and $a \in P$. If $a$ is a cut vertex in $\Gamma(P)$, then $[a]$ is also a cut vertex in $\Gamma_{E}(P)$.

The following example shows that the converse of Lemma 4.2 is not true.

Example 4.3. Let $P_{B}$ be the poset in Figure (B). In $\Gamma_{E}\left(P_{B}\right),\left[a_{1}\right]=\left[a_{2}\right]$ is a cut vertex, since $[b]-\left[a_{1}\right]-[y]$ is the only path from $[b]$ to $[y]$. While, both $b-a_{1}-y$ and $b-a_{2}-y$ are paths from $b$ to $y$ in $\Gamma\left(P_{B}\right)$. Hence, $a_{1}$ is not a cut vertex.

Proposition 4.4. Let $P$ be a poset and $a \in P$. If $[a]$ is a cut vertex in $\Gamma_{E}(P)$, then $[a] \cup\{0\}$ is an ideal of $P$.

Proof. Suppose $b \in[a]$ and $y<b$. We have to show that $y \in[a]$. Since $y<b$, we have that $\operatorname{ann}(b)$ is contained in $\operatorname{ann}(y)$. So $N([a])=N([b])$ is contained in $N([y])$. On the other hand, since $[a]$ is a cut vertex, there exists no vertex $[x]$ distinct from $[a]$ with $N([a])$ containing $N([x])$. Hence, $[y]=[a]$.

Let $P$ be a poset. For $x, y \in P$, if $x$ and $y$ are incomparable, we denote by $y \| x$. For $a \in \operatorname{Atom}(P)$, we define

$\widetilde{U}(\operatorname{Atom}(P) \backslash\{a\})=\{y \in P \mid y \| a$ and $\forall b \in \operatorname{Atom}(P)$, if $b \neq a$, then $y \geq b\}$.

Proposition 4.5. Let $P$ be a poset and $a \in P$. Then $a$ is an atom in $P$ if and only if $[a]$ is an atom in $\bar{P}$ and $a$ is a minimal element in $[a]$.

Proof. $\Rightarrow$ : Suppose $0 \neq[b] \in \bar{P}$ and $[b] \leq[a]$. Then we have ann $(a) \subseteq$ $\operatorname{ann}(b)$. By Proposition 3.9, $\operatorname{ann}(a)$ is maximal in $\operatorname{Anih}(P)$. So we have $\operatorname{ann}(a)=\operatorname{ann}(b)$. That is, $[a]=[b]$. Thus $[a]$ is an atom in $\bar{P}$. Obviously, $a$ is a minimal element in $[a]$.

$\Leftarrow$ : Suppose $0 \neq b \in P$ such that $b \leq a$. We have $\operatorname{ann}(a) \subseteq \operatorname{ann}(b)$, and so $[b] \leq[a]$. Since $[a]$ is an atom in $\bar{P}$, this proves that $[b]=[a]$ or $[b]=[0]$. If $[b]=[0]$, then $b=0$. This is a contradiction. Therefore, we have $[b]=[a]$. Since $a$ is the minimal element in $[a]$, we have $b=a$, and so $a$ is also an atom in $P$. 
Using Proposition 4.5, we have the following theorem characterizing the cut vertices of $\Gamma_{E}(P)$.

Theorem 4.6. Let $P$ be a poset. If $[a] \in \operatorname{Atom}(\bar{P})$ and $a$ is a minimal element in $[a]$, then $[a]$ is a cut vertex in $\Gamma_{E}(P)$ if and only if $\widetilde{U}(\operatorname{Atom}(P) \backslash\{a\}) \neq \emptyset$.

Proof. $\Rightarrow$ : Without loss of generality, let $[x]-[a]-[y]$ be a path of shortest length from $[x]$ to $[y]$. By Lemma 3.3, we have that $x-a-y$ is a path in $\Gamma(P)$. This concludes that $x \| a$ and $y \| a$. If $\widetilde{U}(\operatorname{Atom}(P) \backslash\{a\})=\emptyset$, then we have $u, v \in \operatorname{Atom}(P)$ with $x \| u$ and $y \| v$. If $u \neq v$, then $x-u-v-y$ is a path in $\Gamma(P)$. Using Lemma 3.3 again, we have that $[x]-[u]-[v]-[y]$ is a path in $\Gamma_{E}(P)$. If $u=v$, then $[x]-[u]-[y]$ is a path in $\Gamma_{E}(P)$. In either case, we have a contradiction.

$\Leftarrow:$ If $x \in \widetilde{U}(\operatorname{Atom}(P) \backslash\{a\})$, then $[a]$ is the unique vertex which is adjacent to $[x]$. This proves that $[a]$ is a cut vertex.

In paper [12], Estaji and Khashyarmanesh proved that the clique number of the graph $\Gamma(L)$ is equal to the number of atoms in $L$, where $\Gamma(L)$ is the zerodivisor graph of a lattice $L$ (Theorem 5.13). The following theorem shows that the clique number of the graph $\Gamma_{E}(P)$ is also equal to the number of atoms in $P$.

Theorem 4.7. Let $P$ be a poset. Then $\operatorname{cl}\left(\Gamma_{E}(P)\right)=\mid$ Atom $(P) \mid$.

Proof. By Proposition 4.5, we have $|\operatorname{Atom}(P)|=|\operatorname{Atom}(\bar{P})|$. Since any two atoms in $\bar{P}$ are adjacent, we have $\operatorname{cl}\left(\Gamma_{E}(P)\right) \geq|\operatorname{Atom}(P)|$. Suppose $\left|\operatorname{cl}\left(\Gamma_{E}(P)\right)\right|>|\operatorname{Atom}(P)|$. Let $\operatorname{cl}\left(\Gamma_{E}(P)\right)=m$ and $|\operatorname{Atom}(P)|=n$. Then $\Gamma_{E}(P)$ has a complete subgraph with vertices $\left\{\left[p_{1}\right],\left[p_{2}\right], \ldots,\left[p_{m}\right]\right\}$. Since $\left[p_{i}\right]$ and $\left[p_{j}\right]$ are adjacent in $\Gamma_{E}(P)$, then $\operatorname{atom}\left(p_{i}\right) \cap \operatorname{atom}\left(p_{j}\right)=\emptyset$, for all $i \neq j$. This is impossible, since $m>n$. Hence, $\operatorname{cl}\left(\Gamma_{E}(P)\right)=|\operatorname{Atom}(P)|$.

Let $G$ be a graph and $a, b \in V(G)$. Two vertices $a$ and $b$ are called complements in $G$ if $a$ is connected to $b$, and no vertex in $G$ is connected to both $a$ and $b$, denoted by $a \perp b$. We say that a graph $G$ is complemented if each vertex in $G$ has a complement. The set of all complements in $G$ induces a subgraph of $G$, denoted by $G^{c}$. It is easy to see that $G$ is complemented if and only if $G=G^{c}$. Complements were studied for the zero-divisor graph $\Gamma(R)$ in [3] and for $\Gamma_{E}(R)$ in [2]. The next result is the analog of [2, Theorem $4.3]$.

Proposition 4.8. Let $P$ be a poset. Then the following statements are equivalent.

1) $\Gamma_{E}(P)=\Gamma_{E}(P)^{c}$

2) $\Gamma_{E}(P)$ is complemented.

3) $\Gamma(P)$ is complemented. 
Proof. 1) $\Leftrightarrow 2)$ is obvious.

$2) \Rightarrow 3$ S Suppose $a \in P$ and $[a]$ has a complement $[b]$. Then $[a] \neq[b]$, $[a] \neq[0],[b] \neq[0]$ and $L([a],[b])=\{[0]\}$. Therefore, $a \neq b, a \neq 0, b \neq 0$ and $L(a, b)=\{0\}$ by Lemma 3.3. If there exists a $c \in P$ such that $L(c, a)=$ $L(c, b)=\{0\}$, then $L([c],[a])=L([c],[b])=\{[0]\}$ by Lemma 3.3 and $[c] \notin$ $\{[a],[b]\}$. That is, $[c]$ is adjacent to both $[a]$ and $[b]$. This is a contradiction. Hence $b$ is a complement of $a$ in $\Gamma(P)$.

$3) \Rightarrow 2)$ Suppose $[a] \in V\left(\Gamma_{E}(P)\right)$ and $a \perp b$. Then we have $L([a],[b])=$ $\{[0]\}$. If there exists $[c] \in V\left(\Gamma_{E}(P)\right)$ such that $L([c],[a])=L([c],[b])=\{[0]\}$, then $L(c, a)=L(c, b)=\{0\}$ and $c \notin\{a, b\}$. This is a contradiction. Hence $[a]$ has a complement $[b]$.

Proposition 4.9. Let $P$ be a poset and $\operatorname{Atom}(P)=\left\{a_{1}, a_{2}, \ldots, a_{n}\right\}$. Then

1) $\Gamma(P)$ is an $n$-partite graph.

2) $\Gamma_{E}(P)$ is an n-partite graph.

Proof. 1) Define

$V_{i}=\left\{x \mid x \geq a_{i}\right.$ and if $j<i$, there exists no $a_{j}$ such that $\left.x \geq a_{j}\right\}$.

Then $V_{1}, \ldots, V_{n}$ are disjoint sets and $P \backslash\{0\}=\bigcup_{i=1}^{n} V_{i}$. Suppose $x, y \in V_{i}$, for all $i=1,2, \ldots, n$. Since $x \geq a_{i}$ and $y \geq a_{i}$, there is no edge between $x$ and $y$. Hence, we get the desired result.

2) Let $\overline{V_{i}}=\left\{[x] \mid x \in V_{i}\right\}$. If $[x],[y] \in \overline{V_{i}}$, for all $i=1,2, \ldots, n$, it is easy to see that there is no edge between $[x]$ and $[y]$. So $\Gamma_{E}(P)$ is an $n$-partite graph.

Remark 4.10. Proposition 4.9 can also be obtained directly from [13, Theorem 4.7 and Theorem 2.9].

TheOREM 4.11. Let $P$ be a poset. Then $\Gamma_{E}(P)$ is a complete bipartie graph if and only if $|\operatorname{Atom}(P)|=2$.

Proof. $\Rightarrow$ : Suppose $\Gamma_{E}(P)$ is a complete bipartite graph. If $P$ has only one atom, then $\Gamma_{E}(P)$ is the null graph. Hence, $|\operatorname{Atom}(P)| \geq 2$. If there exist three atoms $a, b, c \in \operatorname{Atom}(P)$, we obviously have a triangle $[a]-[b]-[c]-[a]$. This is impossible, since a complete bipartite graph has no cycle of odd length.

$\Leftarrow$ : Suppose $\operatorname{Atom}(P)=\{a, b\}$. Then $\Gamma_{E}(P)$ is a bipartite graph by Proposition 4.9 .

1) If $x \in P$ such that $x \geq a$ and $x \| b$, then $\operatorname{ann}(x)=\operatorname{ann}(a)$, i.e., $[x]=[a]$.

2) Similarly, if $x \in P$ such that $x \geq b$ and $x \| a$, then $[x]=[b]$.

3 ) If $x \in P$ such that $x \geq a$ and $x \geq b$, then $\operatorname{ann}(x)=\{0\}$, i.e., $[x]=[1]$. In all cases, $\Gamma_{E}(P)$ has two vertices $\{[a],[b]\}$ and so we have $\Gamma_{E}(P)=K_{2}$. 
By the proof of Theorem 4.11, we get the following corollary.

Corollary 4.12. Let $P$ be a poset. Then $\Gamma_{E}(P)=K_{2}$ if and only if $|\operatorname{Atom}(P)|=2$.

Estaji and Khashyarmanesh ([12]) showed that two vertices $a$ and $b$ are adjacent in a zero-divisor graph of a lattice if and only if $\operatorname{atom}(a) \cap \operatorname{atom}(b)=\emptyset$ (Theorem 5.8). The following statement is similar to Theorem 5.8 in [12].

TheOrem 4.13. Let $P$ be a poset. Then

1) $x$ and $y$ are adjacent in $\Gamma(P)$ if and only if atom $(x) \cap$ atom $(y)=\emptyset$.

2) $x$ and $y$ are not adjacent in $\Gamma(P)$ if and only if atom $(x) \cap$ atom $(y) \neq \emptyset$.

Proof. 1) $\Rightarrow$ : If there exists $a \in \operatorname{Atom}(P)$ such that $a \in \operatorname{atom}(x) \cap$ atom $(y)$, then $a \leq x$ and $a \leq y$. This contradicts the fact that $L(x, y)=\{0\}$.

$\Leftarrow$ : Suppose $z \in L(x, y)$. If $z \neq 0$, then there exists an $a \in \operatorname{Atom}(P)$ such that $a \leq z$. Hence, $a \in \operatorname{atom}(x) \cap \operatorname{atom}(y)$. This is a contradiction.

2) By 1), we obviously get 2).

By Theorem 4.13 and Proposition 3.4, we have the following theorem.

TheOREM 4.14. Let $P$ be a poset. Then

1) $[x]$ and $[y]$ are adjacent in $\Gamma_{E}(P)$ if and only if for all $x^{\prime} \in[x]$ and $y^{\prime} \in[y]$, we have atom $\left(x^{\prime}\right) \cap \operatorname{atom}\left(y^{\prime}\right)=\emptyset$.

2) $[x]$ and $[y]$ are not adjacent in $\Gamma_{E}(P)$ if and only if for all $x^{\prime} \in[x]$ and $y^{\prime} \in[y]$, we have atom $\left(x^{\prime}\right) \cap \operatorname{atom}\left(y^{\prime}\right) \neq \emptyset$.

ACKNOWLEDGEMENTS.

The author would like to thank the referees for their careful reviewing and valuable comments which highly improved the paper.

\section{REFERENCES}

[1] M. Alizadeh, A. K. Das, H. R. Maimani, M. R. Pournaki and S. Yassemi, On the diameter and girth of zero-divisor graphs of posets, Discrete Appl. Math. 160 (2012), 1319-1324.

[2] D. F. Anderson and J. D. LaGrange, Commutative Boolean monoids, reduced rings, and the compressed zero-divisor graph, J. Pure Appl. Algebra 216 (2012), 1626-1636.

[3] D. F. Anderson, R. Levy and J. Shapiro, Zero-divisor graphs, von Neumann regular rings, and Boolean algebras, J. Pure Appl. Algebra 180 (2003), 221-241.

[4] D. D. Anderson and M. Naseer, Beck's coloring of a commutative ring, J. Algebra 159 (1993), 500-514.

[5] D. F. Anderson and P. S. Livingston, The zero-divisor graph of a commutative ring, J. Algebra 217 (1999), 434-447.

[6] M. Axtell, N. Baeth and J. Stickles, Cut vertices in zero-divisor graphs of finite commutative rings, Comm. Algebra 39 (2011), 2179-2188.

[7] I. Beck, Coloring of commutative rings, J. Algebra 116 (1988), 208-226.

[8] F. R. DeMeyer and L. DeMeyer, Zero-divisor graphs of semigroups, J. Algebra 283 (2005), 190-198. 
[9] F. R. DeMeyer, T. McKenzie and K. Schneider, The zero-divisor graph of a commutative semigroup, Semigroup Forum 65 (2002), 206-214.

[10] D. Dolžan and P. Oblak, The zero-divisor graph of rings and semirings, Internat. J. Algebra Comput. 22 (2012), 1250033-1-1250033-20.

[11] S. Ebrahimi Atani, An ideal-based zero-divisor graph of a commutative semiring, Glas. Mat. Ser. III 44(64) (2009), 141-153.

[12] E. Estaji and K. Khashyarmanesh, The zero-divisor graph of a lattice, Results Math. 61 (2012), 1-11.

[13] R. Halaš and M. Jukl, On Beck's coloring of posets, Discrete Math. 309 (2009), 4584-4589.

[14] R. Halaš and H. Länger, The zerodivisor graph of a qoset, Order 27 (2010), 343-351.

[15] V. Joshi, Zero divisor graph of a poset with respect to an ideal, Order 29 (2012), 499-506.

[16] D. Lu and T. Wu, The zero-divisor graphs of posets and an application to semigroups, Graphs Combin. 26 (2010), 793-804.

[17] H. R. Maimani, Median and center of zero-divisor graph of commutative semigroups, Iran. J. Math. Sci. Inform. 3 (2008), 69-76.

[18] S. B. Mulay, Cycles and symmetries of zero-divisors, Comm. Algebra 30 (2002), 3533-3558.

[19] S. Spiroff and C. Wickham, A zero divisor graph determined by equivalence classes of zero-divisors, Comm. Algebra 39 (2011), 2338-2348.

[20] Z. Xue and S. Liu, Zero-divisor graphs of partially ordered sets, Appl. Math. Lett. 23 (2010), 449-452

Hongxing Liu

School of Mathematical Sciences

Shandong Normal University

250014 Jinan

P. R. China

E-mail: Ihxshanda@163.com

Received: 5.5.2013.

Revised: 13.11.2013. 